\title{
On Robin's criterion for the Riemann Hypothesis
}

\author{
Safia Aoudjit ${ }^{1}$, Djamel Berkane ${ }^{2}$ and Pierre Dusart ${ }^{3}$ \\ ${ }^{1}$ LAMDA-RO Laboratory, Department of Mathematics, University of Blida1 \\ Po. Box 270 Route de Soumaa, Blida, Algeria \\ e-mail: safiaoudjitg@gmail.com \\ 2 LAMDA-RO Laboratory, Department of Mathematics, University of Blida1 \\ Po. Box 270 Route de Soumaa, Blida, Algeria \\ e-mail: djaber72@univ-blida.dz \\ ${ }^{3}$ Faculté des sciences et techniques, Université de Limoges \\ P.O. Box 123, avenue Albert Thomas 87060 Limoges Cedex, France \\ e-mail: pierre.dusart@unilim.fr
}

Received: 30 November $2020 \quad$ Revised: 15 October $2021 \quad$ Accepted: 8 November 2021

Abstract: Robin's criterion says that the Riemann Hypothesis is equivalent to

$$
\forall n \geq 5041, \quad \frac{\sigma(n)}{n} \leq e^{\gamma} \log _{2} n,
$$

where $\sigma(n)$ is the sum of the divisors of $n, \gamma$ represents the Euler-Mascheroni constant, and $\log _{i}$ denotes the $i$-fold iterated logarithm. In this note we get the following better effective estimates:

$$
\forall n \geq 3, \frac{\sigma(n)}{n} \leq e^{\gamma} \log _{2} n+\frac{0.3741}{\log _{2}^{2} n} .
$$

The idea employed will lead us to a possible new reformulation of the Riemann Hypothesis in terms of arithmetic functions.

Keywords: Primorial number, Robin's inequality, Riemann Hypothesis.

2020 Mathematics Subject Classification: 11A25, 11N64, 11M26.

\section{Introduction and statement of results}

As usual, let $\left(p_{k}\right)_{k \geq 1}$ denote the increasing sequence of prime numbers, and let $N_{k}$ be the primorial integer of index $k$, the product of its $k$ first terms. The Riemann Hypothesis (RH) claims that the 
nontrivial zeros of zeta function $\zeta(s)=\sum_{n \geq 1} n^{-s}$ are located on the critical line $\mathcal{R}(s)=\frac{1}{2}$. Several equivalent formulations of RH appeared, but the one which interests us here is that in terms of arithmetic functions, here we cite the first papers of Gronwall [8], Nicolas [11] and Robin [13], followed by, for instance, Akbary [1], Caveney et al. [6] and Lagarias [10].

Robin in his paper [13] asserted that RH is equivalent to

$$
\forall n \geq 5041, \quad \sigma(n) \leq e^{\gamma} n \log _{2} n,
$$

with $\sigma(n)$ denotes the sum of divisors function, $\gamma$ the Euler-Mascheroni constant, and $\log _{i}$ the $i$-fold iterated logarithm. This assertion is based on the known following formula (see [9]):

$$
\frac{\sigma(n)}{n}=(1+o(1)) e^{\gamma} \log _{2} n .
$$

In this note, we intend to join the authors who have attempted to closely determine the $o$-term in the formula (2). The best upper bound of the normalized of the sum of divisors function is also given by Robin [13] which proved, unconditionally, that

$$
\forall n \geq 3, \frac{\sigma(n)}{n} \leq e^{\gamma} \log _{2} n+\frac{0.6483}{\log _{2} n} .
$$

We propose the following result:

Theorem 1.1. For every integer $n \geq 3$, we have

$$
\frac{\sigma(n)}{n} \leq e^{\gamma} \log _{2} n+\frac{0.3741}{\log _{2}^{2} n}
$$

This improves considerably Robin's upper bound. In parallel, we study another form of upper bound than that exposed in the theorem above, since it is completely expressed in terms of $K(x)$, the primorial counting function which, see Balazard [4], is approximately $\frac{\log x}{\log _{2} x}$. We conclude that:

Theorem 1.2. If $K(n)$ is the number of primorial integers not exceeding $n$, then

$$
\forall n \geq 30, \frac{\sigma(n)}{n} \leq e^{\gamma}\left(\log K(n)+\log _{2} K(n)+\frac{\log _{2} K(n)}{\log K(n)}+\frac{1}{20 \log _{2}^{2} K(n)}\right) .
$$

This leads us to examine a conjecture upon which we stumbled:

Conjecture 1. The Riemann Hypothesis is equivalent to

$$
\forall n \geq 205, \frac{\sigma(n)}{n} \leq e^{\gamma}\left(\log K(n)+\log _{2} K(n)+\frac{\log _{2} K(n)}{\log K(n)}\right) .
$$

See Section 4 for more background on this conjecture. The main ingredient of this paper is the recent version of the upper bound of the product over primes $\prod_{p \leq x} \frac{p}{p-1}$, thanks to the paper of the third author in [7], as a consequence of the new estimates of Chebyshev's summatory functions also exposed in [7]. Although there are some updates, such improvements have negligible influence on the final results. Finally, we indicate that $e$ represents Napier's constant, $p$ a prime number, and with this technique, obtaining better approximations is closely linked with progress on extending the known zero-free region of the Riemann zeta-function. 


\section{Preliminary lemmas}

The primorial counting function $K(x)$ is not known in the literature. We begin by showing some basic properties (for a more extended study, see the recent paper of the authors [3]). For each real $x \geq 1$, the integer $K(x)$ can be defined by $\max \left\{k \in \mathbb{N}^{*}, N_{k} \leq x\right\}$. In the following lemma, we prove that for a given $x \geq 1$, the primorial $N_{K(x)}$ represent the smallest integer less than $x$ whose decomposition into prime numbers is the longest. Here $\omega(n)$ denotes the number of prime distinct divisors of $n$.

Lemma 2.1. For every real number $x \geq 1$, we have

$$
K(x)=\max _{1 \leq n \leq x} \omega(n) .
$$

Furthermore, for any integer $n \leq x$ with $\omega(n)=K$, we have $N_{K} \leq n$.

Proof. As $N_{k} \leq n \leq x<N_{k+1}$ means that $\omega(n) \leq k$ and $K(n)=k$, hence $\omega(n) \leq K(n)$ in any interval $\left[N_{k}, N_{k+1}[\right.$, which implies that

$$
\max _{1 \leq n \leq x} \omega(n)=\max _{1 \leq n<N_{K+1}} \omega(n)=K .
$$

Let $q_{1} q_{2} \cdots q_{K}$ be an integer less than $x$ with $q_{1}<q_{2}<\cdots<q_{K}$ prime numbers. For $K=1$ it is obvious that $q_{1} \geq p_{1}$. Now, assuming $q_{i} \geq p_{i}$ for $i<K$, it is necessary that $q_{K} \geq p_{K}$, otherwise $q_{K}<q_{K-1}$.

Lemma 2.2. We have, when $x \geq 8$, the following inequalities:

$$
\log _{2} x<K(x) \leq \log x
$$

Proof. From the definition of $K(x)$, by taking the logarithm, we can also write the following:

$$
K(x)=\max \left\{k \in \mathbb{N}^{*}, \theta\left(p_{k}\right) \leq \log x\right\},
$$

where $\theta$ denotes the Chebyshev function. So, by recalling the inequality $\theta\left(p_{k}\right) \geq k$ given in Robin [12] valid once $k \geq 3$, one easily deduces that

$$
K(x) \leq \max \left\{k \in \mathbb{N}^{*}, k \leq \log x\right\} \leq \log x, \forall x \geq N_{3},
$$

which is also valid for $8 \leq x<N_{3}$. For the second, a short induction on $k$ is necessary. For all $k \geq 1$, we have $N_{k}<e^{e^{k-1}}$. Indeed, the case $k=1$ is obvious, and the fact that $\forall k \geq 1, p_{k+1}<N_{k}$ (according to Euclid's proof of the infinity of primes) implies that

$$
N_{k+1}=N_{k} p_{k+1}<e^{e^{k-1}} N_{k}<e^{2 e^{k-1}}<e^{e e^{k-1}}=e^{e^{k}} .
$$

So, by taking the logarithm, one gets that for all $x \geq e$ :

$$
\log _{2} x<\log _{2} N_{K+1}<K(x) .
$$

We conclude the proof using computer verifications for the small values. In relation to $\pi(x)$ the prime counting function, we can also mention that

$$
\log _{2} x<K(x) \leq \log x<\pi(x) .
$$


Lemma 2.3. Let $\delta=1.000081$. We have, when $x \geq 210$ :

$$
K(x) \geq \frac{1}{\delta} \frac{\log x}{\log _{2} x} .
$$

Proof. Recalling the following estimates given in [14]:

$$
\theta(x)<\delta x, \forall x>1 \text { and } \pi(x) \geq \frac{x}{\log x}, \forall x \geq 17,
$$

one reaches successively, for every real $x \geq e^{17 \delta}$, that

$$
K(x) \geq \max \left\{k \in \mathbb{N}^{*}, \delta p_{k} \leq \log x\right\}=\pi\left(\frac{\log x}{\delta}\right) \geq \frac{1}{\delta} \frac{\log x}{\log _{2} x} .
$$

A computer check handles the cases $210 \leq x<e^{17 \delta}$.

Now, for $f$ a decreasing function greater than 1 on $(1, \infty)$, we consider the following sequence

$$
\mathfrak{L}(n)=\prod_{p \mid n} f(p), \forall n>1 .
$$

The term $\mathfrak{L}(n)$ for the function $f(x)=\frac{x}{x-1}$ is only $\frac{n}{\varphi(n)}$, where $\varphi(n)$ denotes the Euler totient function, and $\mathfrak{L}(n)$ is $\frac{\Psi_{t}(n)}{n}$ when $f(x)=1+1 / x+\cdots+1 / x^{t-1}, t \geq 2$, where $\Psi_{t}(n)$ is the generalized Dedekind psi function. We have the following Lemmas

Lemma 2.4. For every real number $x \geq 2$, the following equality

$$
\max _{1<n \leq x} \mathfrak{L}(n)=\prod_{p \leq p_{K(x)}} f(p)
$$

holds.

Proof. To determine the maximum of $\mathfrak{L}(n)$, when $n$ range over all integers less than or equal to $x$, we first use the fact that $f$ is greater than 1 since this places the maximum at the class of the integers whose number of prime divisors is the largest. Then, as $f$ is also strictly decreasing, the maximum must have the smallest prime numbers in its decomposition. However, according to the previous lemma, we can clearly specify that, it is only true for $N_{K(x)}$, i.e.,

$$
\max _{1<n \leq x} \mathfrak{L}(n)=\mathfrak{L}\left(N_{K(x)}\right) .
$$

Finally, as $p \mid N_{k}$ is equivalent to $p \leq p_{k}$, the lemma follows.

Remark 1. When $f$ is strictly increasing and greater than 1 on $(1, \infty)$, the maximum of $\mathfrak{L}(n)$ is reached at an integer $q_{1} \cdots q_{K(x)}$, where at least one of $q_{i}$ is a prime number greater than $p_{i}$.

In the following lemma, we leave the generalization and show, through a simpler proof, a result concerning the order of the Euler function.

Lemma 2.5. We have

$$
\limsup _{n \rightarrow+\infty} \frac{n}{e^{\gamma} \varphi(n) \log _{2} n}=1
$$


Proof. From the previous lemma and the definition of $K(n)$, we deduce that

$$
\frac{\mathfrak{L}(n)}{\log _{2} n} \leq \frac{\mathfrak{L}\left(N_{K(n)}\right)}{\log _{2} N_{K(n)}}
$$

So, our limit becomes as follows:

$$
\limsup _{n \rightarrow+\infty} \frac{\mathfrak{L}(n)}{e^{\gamma} n \log _{2} n}=\lim _{k \rightarrow+\infty} \frac{\mathfrak{L}\left(N_{k}\right)}{e^{\gamma} N_{k} \log _{2} N_{k}} .
$$

In particular, when $f(x)=\frac{x}{x-1}$, one obtains according to Mertens' theorem that

$$
\mathfrak{L}\left(N_{k}\right)=\prod_{p \leq p_{k}} \frac{p}{p-1} \sim e^{\gamma} \log p_{k},
$$

as $k \rightarrow+\infty$. Thus, the lemma follows by recalling that

$$
\log _{2} N_{k}=\log \left(\theta\left(p_{k}\right)\right) \sim \log p_{k}
$$

using the Prime Number Theorem.

Every proof containing explicit results requires at some point or another a digital verification of the property obtained on the finite number of cases that remain. In our case, we need to compute the values of $\frac{\sigma(n)}{e^{\gamma} n \log _{2} n}$ for fairly large $n$. We will use the result of Briggs [5], where he checked Robin's inequality up to $10^{10^{10}}$.

Lemma 2.6 (Briggs). Robin's criterion holds, for $5040<n \leq 10^{10^{10}}$.

We end this section by mentioning the following recent explicit bounds of $\theta(x)$ and $\prod_{p \leq x}\left(1-\frac{1}{p}\right)$. Lemma 2.7 (Dusart). The following estimates hold

$$
\begin{gathered}
\theta(x) \geq x\left(1-\frac{0.01}{\log ^{3} x}\right), \text { as soon as } x \geq 7232121212 . \\
\prod_{p \leq x}\left(1-\frac{1}{p}\right)^{-1} \leq e^{\gamma} \log x\left(1+\frac{0.2}{\log ^{3} x}\right), \text { when } x \geq 2278382 . \\
\theta\left(p_{k}\right) \geq k\left(\log k+\log _{2} k-1+\frac{\log _{2} k-2.050735}{\log k}\right), \text { when } p_{k} \geq 10^{11} . \\
p_{k} \leq k\left(\log k+\log _{2} k-1+\frac{\log _{2} k-1.95}{\log k}\right), \text { when } k \geq 178974
\end{gathered}
$$

\section{Proof of Theorem 1.1}

To begin with, for $n$ such that $K:=K(n) \geq K_{1}=164607$ we have $p_{K} \geq 2228382$. This implies by Lemmas $[2.4,2.7]$ that

$$
\frac{n}{\varphi(n)} \leq \prod_{p \leq p_{K}} \frac{p}{p-1} \leq e^{\gamma} \log p_{K}\left(1+\frac{0.2}{\log ^{3} p_{K}}\right) .
$$

On the other hand, according to inequality (4), once $K \geq K_{2}=7232121212$, it follows that

$$
\log _{2} N_{K}=\log \theta\left(p_{K}\right) \geq \log p_{K}-\frac{0.01}{\log ^{3} p_{K}} .
$$


Now, with some care, one can write for $K \geq K_{2}$ the following

$$
\begin{aligned}
e^{\gamma} \log p_{K}\left(1+\frac{0.2}{\log ^{3} p_{K}}\right) & =e^{\gamma} \log p_{K}+\frac{0.2 e^{\gamma}}{\log ^{2} p_{K}} \\
& =e^{\gamma} \log p_{K}\left(1-\frac{0.01}{\log ^{2} p_{K}}\right)+\frac{(0.2+0.01) e^{\gamma}}{\log ^{2} p_{K}} \\
& =e^{\gamma} \log p_{K}\left(1-\frac{0.01}{\log ^{3} p_{K}}\right)+\frac{0.3741}{\log ^{2} p_{K}}
\end{aligned}
$$

Hence, taking into account that the function $e^{\gamma} t+\frac{0.3741}{t^{2}}$ is increasing for $t \geq 1$, we easily deduce from inequality (9) that

$$
e^{\gamma}\left(\log p_{K}-\frac{0.01}{\log ^{2} p_{K}}\right)+\frac{0.3741}{\log ^{2} p_{K}}<e^{\gamma} \log _{2} N_{K}+\frac{0.3741}{\log _{2}^{2} N_{K}}
$$

and then

$$
\frac{n}{\varphi(n)} \leq e^{\gamma} \log _{2} N_{K}+\frac{0.3741}{\log _{2}^{2} N_{K}}, \forall K \geq K_{2} .
$$

By computer, the last inequality is shown to be also valid when $2 \leq K<K_{2}$. Consequently, invoking again the increase of the function $e^{\gamma} t+\frac{0.3741}{t^{2}}$, one gets for $n \geq N_{2}$, and then for $n \geq 3$ that

$$
\frac{n}{\varphi(n)} \leq e^{\gamma} \log _{2} n+\frac{0.3741}{\log _{2}^{2} n}
$$
follows.

Finally, as the inequality $\frac{\sigma(n)}{n} \leq \frac{n}{\varphi(n)}$ holds (see [13, page 193]) for $n \geq 1$, the theorem

The following direct consequence joins the upper bounds of $\frac{\sigma(n)}{n}$ in the form $(1+\epsilon) e^{\gamma} \log _{2} n$ given in [2] for different values of $\epsilon$. The value $\epsilon=0.0000123$ obtained below, once $n \geq 5041$, remains stable until the best value $\epsilon=0.005558981 \ldots$ obtained in [2], as soon as $n \geq 2521$.

Corollary 3.1. For every integer $n \geq 5041$, we have

$$
\frac{\sigma(n)}{n} \leq(1.0000123) e^{\gamma} \log _{2} n .
$$

Proof. The idea is to take the term $\frac{0.3741}{\log _{2}^{2} n}$ from Theorem 1.1, divide it by $e^{\gamma} \log _{2} n$, then calculate the image of $10^{10^{10}}$. The remainder is guaranteed by Lemma 2.6.

\section{Proof of Theorem 1.2}

By inequality (5) we infer that for every $k \geq K_{1}=164607$ :

$$
\frac{N_{k}}{\varphi\left(N_{k}\right)}=\prod_{p \leq p_{k}} \frac{p}{p-1} \leq e^{\gamma} \log p_{k}\left(1+\frac{0.2}{\log ^{3} p_{k}}\right) .
$$

However; see [12], we have

$$
k \log k \leq p_{k} \leq k\left(\log k+\log _{2} k\right),
$$


once $k \geq 6$. So, we obtain the following inequalities:

$$
2 \log _{2} k \leq \log p_{k} \leq \log k+\log _{2} k+\frac{\log _{2} k}{\log k}, \forall k \geq 6
$$

which implies successively for $k \geq K_{1}$ :

$$
\begin{aligned}
\frac{N_{k}}{\varphi\left(N_{k}\right)} & \leq e^{\gamma}\left(\log p_{k}+\frac{0.2}{\log ^{2} p_{k}}\right) \\
& \leq e^{\gamma}\left(\log k+\log _{2} k+\frac{\log _{2} k}{\log k}+\frac{0.2}{4 \log _{2}^{2} k}\right) .
\end{aligned}
$$

Then, it comes by computer that the last upper bound also holds for $k \geq 10$. Hence, one gets for all $n \geq N_{10}$, according to Lemma 2.4 , that

$$
\frac{n}{\varphi(n)} \leq e^{\gamma}\left(\log K(n)+\log _{2} K(n)+\frac{\log _{2} K(n)}{\log K(n)}+\frac{0.2}{4 \log _{2}^{2} K(n)}\right) .
$$

Now, let us go back to the ratio $\frac{\sigma(n)}{n}$. According to [13], this quantity takes maximal values on so called colossally abundant (CA) numbers, and if Robin's inequality is true on consecutive CA numbers $C A_{i}$ and $C A_{i+1}$, then it is also true for all integer $n \in\left[C A_{i}, C A_{i+1}\right]$. We say that $n$ is colossally abundant if there exists a positive $\epsilon$ for which:

$$
\frac{\sigma(n)}{n^{1+\epsilon}} \geq \frac{\sigma(k)}{k^{1+\epsilon}}, \forall k>1
$$

Thus, to complete our proof, it suffices to check inequality (10) for $\frac{\sigma(n)}{n}$ only on the CA numbers less than $N_{10}$, namely: 2, 6, 12, 60, 120, 360, 2520, 5040, 55440, 720720, 1441440, 4324320, 21621600,367567200 and 6983776800 .

Next, this leads us to discuss a possible reformulation of RH in terms of arithmetic functions. First, we observe that the following proposition

Proposition 1. We have, when $205 \leq n \leq C A_{160}$, the inequality

$$
\frac{\sigma(n)}{n} \leq e^{\gamma}\left(\log K(n)+\log _{2} K(n)+\frac{\log _{2} K(n)}{\log K(n)}\right),
$$

where $C A_{160}>10^{326}$.

Proof. It suffices to check the list of terms of the sequence registered as A004490 of CA numbers in OEIS [15]. This extends the inequality to all integers between 205 and $C A_{160}$.

The following table shows part of the calculations, where $e^{\gamma} A(n)$ is the upper bound of Proposition 1. 


\begin{tabular}{|c|c|c|c|}
\hline $\boldsymbol{n}$ & $\boldsymbol{\sigma}(\boldsymbol{n}) / \boldsymbol{n}$ & $\boldsymbol{K}(\boldsymbol{n})$ & $\boldsymbol{e}^{\gamma} \boldsymbol{A}(\boldsymbol{n})-\boldsymbol{\sigma}(\boldsymbol{n}) / \boldsymbol{n}$ \\
\hline \hline$C A_{150}=N_{121} N_{11} N_{5} N_{3} N_{2}^{3} N_{1}^{4}$ & 11.570817 & 127 & 0.44727552 \\
\hline$C A_{151}=N_{122} N_{11} N_{5} N_{3} N_{2}^{3} N_{1}^{4}$ & 11.588010 & 128 & 0.44658941 \\
\hline$C A_{152}=N_{123} N_{11} N_{5} N_{3} N_{2}^{3} N_{1}^{4}$ & 11.605127 & 129 & 0.44584657 \\
\hline$C A_{153}=N_{124} N_{11} N_{5} N_{3} N_{2}^{3} N_{1}^{4}$ & 11.622118 & 130 & 0.44509823 \\
\hline$C A_{154}=N_{125} N_{11} N_{5} N_{3} N_{2}^{3} N_{1}^{4}$ & 11.638937 & 131 & 0.44439327 \\
\hline$C A_{155}=N_{126} N_{11} N_{5} N_{3} N_{2}^{3} N_{1}^{4}$ & 11.655541 & 132 & 0.44377752 \\
\hline$C A_{156}=N_{127} N_{11} N_{5} N_{3} N_{2}^{3} N_{1}^{4}$ & 11.671980 & 133 & 0.44320089 \\
\hline$C A_{157}=N_{128} N_{11} N_{5} N_{3} N_{2}^{3} N_{1}^{4}$ & 11.688214 & 134 & 0.44270719 \\
\hline$C A_{158}=N_{129} N_{11} N_{5} N_{3} N_{2}^{3} N_{1}^{4}$ & 11.704291 & 135 & 0.44224879 \\
\hline$C A_{159}=N_{130} N_{11} N_{5} N_{3} N_{2}^{3} N_{1}^{4}$ & 11.720259 & 136 & 0.44178089 \\
\hline$C A_{160}=N_{131} N_{11} N_{5} N_{3} N_{2}^{3} N_{1}^{4}$ & 11.736118 & 137 & 0.44130365 \\
\hline
\end{tabular}

This completes the proof.

In view of this numerical experiments the natural question is:

Question 1. Is it true that

$$
\frac{\sigma(n)}{n} \leq e^{\gamma}\left(\log K(n)+\log _{2} K(n)+\frac{\log _{2} K(n)}{\log K(n)}\right),
$$

for all $n \geq 205$ ?

An answer to this question is linked to $\mathrm{RH}$ by the following proposition:

Proposition 2. If the Riemann Hypothesis hold, we have for every integer $n \geq 205$ :

$$
\frac{\sigma(n)}{n} \leq e^{\gamma}\left(\log K(n)+\log _{2} K(n)+\frac{\log _{2} K(n)}{\log K(n)}\right) .
$$

Proof. This is deduced from Robin's criterion and essentially from the fact that $A(n) \geq \log _{2} n$, for every $n \geq 10^{322}$. Indeed, one gets from Lemma 2.3 that

$$
\begin{gathered}
\log K(x) \geq \log _{2} x-\log _{3} x-\log \delta, \forall x \geq 3, \\
\log _{2} K(x) \geq \log _{3} x+\log \left(1-\frac{\log _{3} x+\log \delta}{\log _{2} x}\right), \forall x \geq 3,
\end{gathered}
$$

and from Lemma 2.2 the following

$$
\frac{\log _{2} K(x)}{\log K(x)} \geq \frac{\log _{4} x}{\log _{3} x}, \forall x \geq 15
$$

Thus, inequalities (11), (12) and (13) yield us for $x \geq 15$ :

$$
A(x) \geq \log _{2} x+\frac{\log _{4} x}{\log _{3} x}+\log \left(1-\frac{\log _{3} x+\log \delta}{\log _{2} x}\right)-\log \delta .
$$

By setting $\log _{2} x=t$, the study of the following function:

$$
\frac{\log _{4} x}{\log _{3} x}+\log \left(1-\frac{\log _{3} x+\log \delta}{\log _{2} x}\right)-\log \delta
$$

becomes less complicated, and reveals that it is increasing and positive as soon as $x \geq 10^{322}$. 
This implies that

$$
A(x) \geq \log _{2} x, \forall x \geq 10^{322} .
$$

Finally, if the Riemann Hypothesis holds, first we have from Robin's criterion that $\frac{\sigma(n)}{n} \leq e^{\gamma} A(n)$ for all $n \geq 10^{322}$, and thanks to the computations of Proposition 1 for the remaining values.

At this level, part of Conjecture 1 is proven and the persistent question is:

Question 2. Is it true that if $R H$ is false, the inequality

$$
\frac{\sigma(n)}{n} \leq e^{\gamma}\left(\log K(n)+\log _{2} K(n)+\frac{\log _{2} K(n)}{\log K(n)}\right)
$$

is violated for infinitely many $n \geq N_{3}$ ?

A heuristic motivation runs as follows:

$$
\begin{gathered}
K(n) \approx \log n / \log _{2} n \underset{\log n / \log _{2} n \rightarrow 1}{\Longrightarrow} \log K(n) \approx \log _{2} n-\log _{3} n \approx \log _{2} n \\
\Longrightarrow \log K(n)+\log _{2} K(n) \approx \log _{2} n \\
\Longrightarrow A(n) \approx \log _{2} n .
\end{gathered}
$$

Hence, according to Robin's criterion, since $\frac{\sigma(n)}{n}>e^{\gamma} \log _{2} n$ infinitely often, if the Riemann Hypothesis is false, as $A(n) \approx \log _{2} n$, there may exist infinitely many $n$ such that

$$
\frac{\sigma(n)}{n}>e^{\gamma} A(n) \text {. }
$$

\section{Acknowledgements}

The authors wish to thank the referees for their constructive comments and suggestions.

\section{References}

[1] Akbary, A., \& Francis, F. J. (2020). Euler's function on products of primes in a fixed arithmetic progression. Mathematics of Computation, 89(322), 993-1026.

[2] Akbary, A., \& Friggstad, Z. (2009). Superabundant numbers and the Riemann Hypothesis. American Mathematical Monthly, 116(3), 273-275.

[3] Aoudjit, S., Berkane, D., \& Dusart, P. (2021). Explicit estimates involving the primorial integers and applications. Journal of Integer Sequences, 24(7), Article 21.7.8.

[4] Balazard, M. (1990). Unimodalité de la distribution du nombre de diviseurs premiers d'un entier. Annales de l'Institut Fourier, 2, 255-270.

[5] Briggs, K. (2006). Abundant numbers and the Riemann Hypothesis. Experimental Mathematics, 15 (2), 251-256. 
[6] Caveney, G., Nicolas, J. L., \& Sondow, J. (2011). Robin's theorem, primes, and a new elementary reformulation of the Riemann Hypothesis. Integers, 11(6), 753-763.

[7] Dusart, P. (2018). Estimates of the $k$-th prime under the Riemann hypothesis. The Ramanujan Journal, 47(1), 141-154.

[8] Gronwall, T. H. (1913). Some asymptotic expressions in the theory of numbers. Transactions of the American Mathematical Society, 14, 113-122.

[9] Hardy, G. H., \& Wright, E. M. (1960). An Introduction to the Theory of Numbers. 4th ed. Oxford University Press.

[10] Lagarias, J. C. (2002). An Elementary Problem Equivalent to the Riemann Hypothesis. American Mathematical Monthly, 109, 534-543.

[11] Nicolas, J. L. (1983). Petites valeurs de la fonction d'Euler. Journal of Number Theory, 17, $375-388$.

[12] Robin, G. (1983). Estimation de la fonction de Tchebychef $\theta$ sur le $k^{\text {ième }}$ nombre premier et grande valeurs de la fonction $\omega(n)$ nombre de diviseurs premiers de $n$. Acta Arithmetica, $42,367-389$.

[13] Robin, G. (1984). Grandes valeurs de la fonction somme des diviseurs et hypothèse de Riemann. Journal de Mathématiques Pures et Appliquées. Neuvième Série, 63, 187-213.

[14] Rosser, J. B., \& Schoenfeld, L. (1962). Approximate formula for the some functions of Prime Numbers. Illinois Journal of Mathematics, 6, 64-94.

[15] Sloane, N. J. A. A004490. The On-Line Encyclopedia of Integer Sequences. Available online at: https: / / oeis.org/A004490. 